EPJ Web of Conferences 21, 04006 (2012)

DOI: $10.1051 /$ epjconf/20122104006

(C) Owned by the authors, published by EDP Sciences, 2012

\title{
Investigation of dipole strength at the ELBE accelerator in Dresden-Rossendorf
}

\author{
R. Massarczyk ${ }^{12, a}$, G. Schramm ${ }^{12}$, E. Birgersson ${ }^{1}$, R. Schwengner ${ }^{1}$, E. Grosse ${ }^{12}$, A.R. Junghans ${ }^{1}$, \\ and A. Wagner ${ }^{1}$ \\ 1 Institute for Radiation Physics, Helmholtz-Zentrum Dresden-Rossendorf, Germany \\ 2 Institute for Nuclear and Particle Physics, Technische Universität Dresden, Germany
}

\begin{abstract}
Using the methods of cold neutron capture and photon scattering the electric dipole strength function and the level density of the nuclei ${ }^{78} \mathrm{Se}$ and ${ }^{196} \mathrm{Pt}$ are investigated. Considering that the deexcitation process could be described by the same strength functions one could describe both experiments in a statistical model code. The report shows the data analysis as well as a new very fast statistical code, which was used to get the complete strength information up to the neutron separation energy.
\end{abstract}

\section{Introduction}

The electric dipole strength of nuclei has a large resonance at excitation energies of about $10-30 \mathrm{MeV}$, the well known giant dipole resonance (GDR). Different models exist to parametrize this structure. One approach is to fit known $(\gamma, \mathrm{n})$ cross sections with one or two Lorentzian functions as used in the RIPL3 database [1]. Another more general approach is used by the Triple-Lorentzian-model [2].

These approaches have the aim to give over a wide range of nuclei an easy parametrization of the photo-nuclear cross section, which is needed for a better understanding in many applications, from providing a database for the construction of the new Generation IV power plants, over the use in network calculations of astrophysical scenarios [3], up to a comparison of theoretical predictions like RPA [4].

Since a few years the low energy part of these distributions became more and more interesting because one the one hand side this is the region, where the different models differ a lot and no data is available as it could be seen in fig. 1. On the other hand several experiments [6-8] have shown some extra strength in this region, often called pygmy resonance. The method of nuclear resonance fluorescence (NRF) has been proved in several experiments to be a tool to determine the photo absorption cross section up to the neutron separation energy. The bremsstrahlung facility [9] at the superconducting electron accelerator ELBE of the Helmholtz-Zentrum Dresden-Rossendorf opens up the possibility to study this interesting energy region.

The research reactor at the IKI Budapest can be used to provide cold neutrons for capture experiments. Both facilities and the data analysis of both experiments will be shown in the following chapter in a short overview.

The idea of this twin experiment was to compare the deexcitation of excited states in the same nuclei with different methods of excitation. In order to guarantee equal conditions, two stable nuclei are needed, one with ground state spin and parity $\mathrm{J}^{P}=\frac{1}{2}^{-}$for neutron capture and one with $\mathrm{J}^{P}=0^{+}$for the scattering experiment. The capture of an s-wave neutron by an nucleus with mass A produces an A +1 excited nuclei with with predominantly $1^{-}$states. This is also the case for the dipole excitation of the $\mathrm{A}+1$ nuclei from ground state $0^{+}$to $\mathrm{J}^{P}=1^{-}$. Not many nuclei in the nuclide chart fulfill these conditions. We used ${ }^{77} \mathrm{Se}$ and ${ }^{78} \mathrm{Se}$ as well as ${ }^{195} \mathrm{Pt}$ and ${ }^{196} \mathrm{Pt}$ for this twin experiment.

\footnotetext{
a e-mail: r.massarczyk@hzdr.de
}

This is an Open Access article distributed under the terms of the Creative Commons Attribution-Noncommercial License 3.0, which permits unrestricted use, distribution, and reproduction in any noncommercial medium, provided the original work is properly cited. 
${ }^{78}$ Se photo absorption cross section

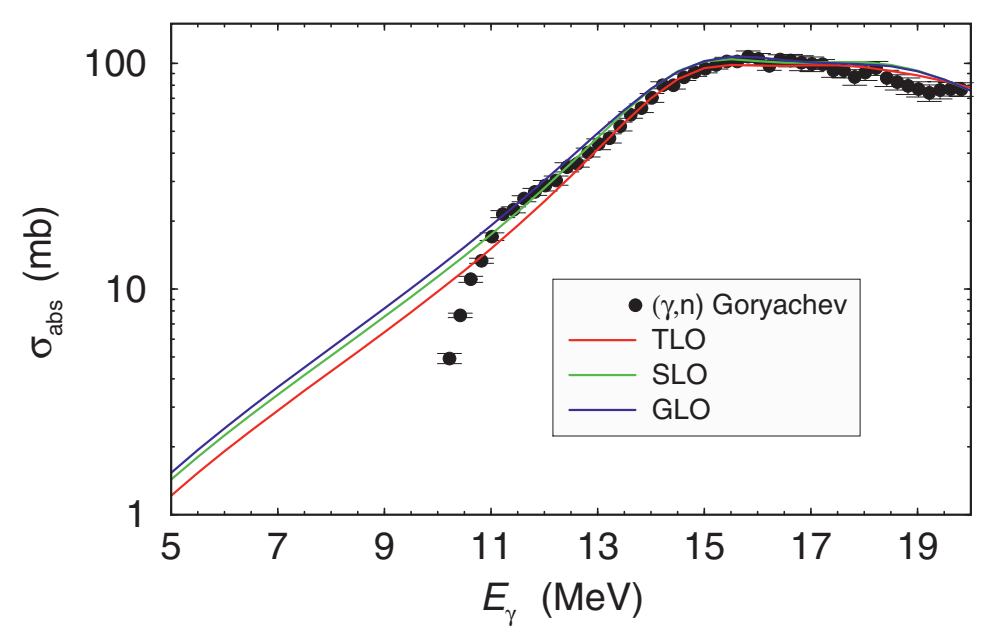

Fig. 1. Photo-absorption cross section for ${ }^{78} \mathrm{Se}$. Different models are shown as mentioned in the text as well as $(\gamma, \mathrm{n})$ data from reference [5].

After the measurement different simulations have to be performed to understand background events and detector response. For this purpose the GEANT4 [10] package was used. A new statistical code was developed [11] to understand the shape of the observed spectra and to determine essential input parameters like level density.

This work is part of an actual paper, which contains a closer view to all details [12].

\section{$2(\mathbf{n}, \gamma)$ at IKI Budapest}

\subsection{Experimental site}

At the Budapest research reactor the first part of this twin experiment took place. At $10 \mathrm{MW}$ thermal power the neutron flux in the cold energy region is in the order of $5 \cdot 10^{7}$ neutrons $/\left(\mathrm{cm}^{2} \cdot \mathrm{s}\right)$ at the target position which has been at the PGAA (prompt gamma activation analysis) station [13]. A 99.6\% enriched ${ }^{77} \mathrm{Se}$ target was used. After the capture of a cold neutron the excited compound nucleus ${ }^{78} \mathrm{Se}$ is formed. This decays via one or more steps back to the ground state.

An energy spectrum of the emitted $\gamma$-rays was collected with a high-purity germanium (HPGe) detector, which was brought from Dresden to Budapest to ensure equal conditions in photon detection. The detector system includes a surrounding bismuth germanate (BGO) scintillation detector which is used as an escape suppression shield.

In fig. 2 is shown how a spectra, in this case a test measurement with deuterated urea, changes with the active BGO-Veto and after removing the detector response simulated with GEANT4. After the correction the spectra consists only full energy peaks. The same algorithm for correcting the spectra was used for the neutron capture of ${ }^{77} \mathrm{Se}$. In fig. 3 one can see, a broad continuum containing many unresolved peaks. This cleaned spectra is now input for a statistical analysis to deduce informations about the nuclear level density and the strength function. 
$\mathrm{CNR} * 11$

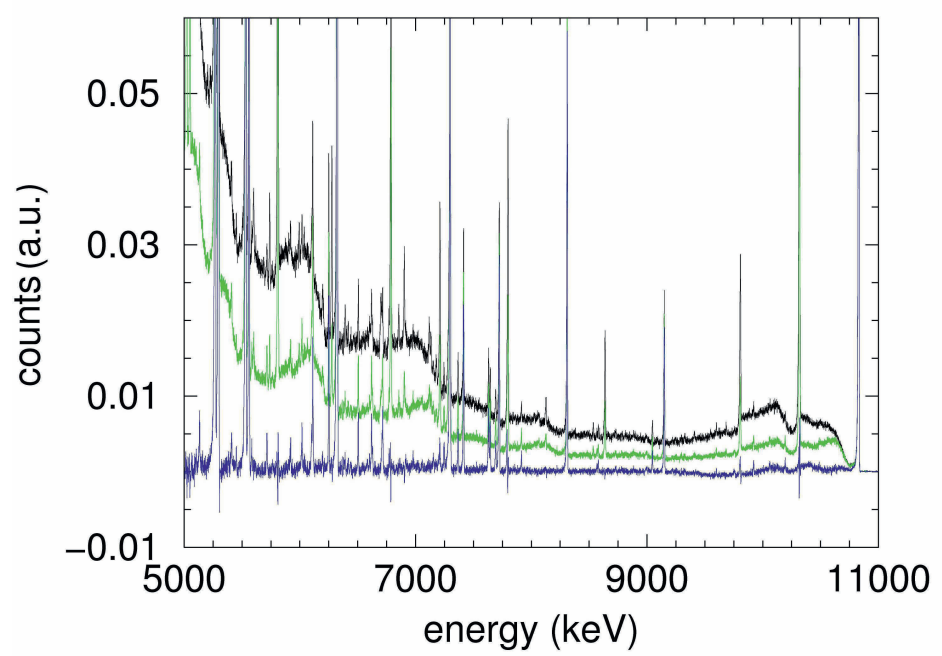

Fig. 2. spectra of neutron capture in nitrogen, without BGO-Veto (black), with BGO-Veto (green), after correction of detector response (blue)

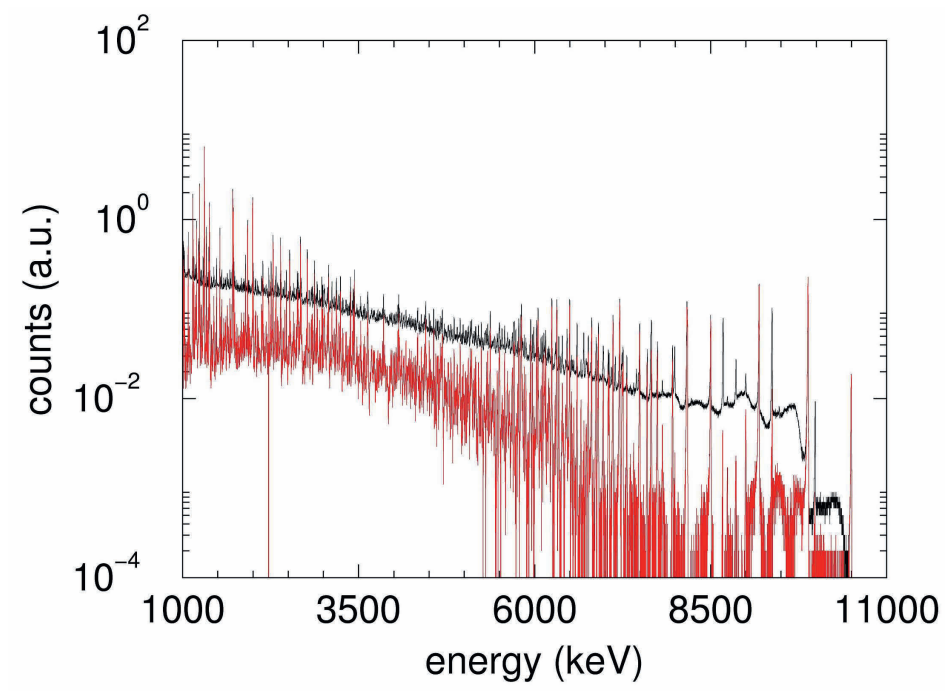

Fig. 3. spectra of neutron capture in ${ }^{77} \mathrm{Se}$. The original spectrum (black) is corrected for background and detector response (red)

\subsection{Statistical analysis}

The excited state has different possibilities to deexcite. It could decay directly to the ground state or it can decay to a lower lying state. In fig. 3 one clearly sees the decay to the ground state, which is the large peak at $10.49 \mathrm{MeV}$. Below this one can observe also strong transitions to the first excited states. But there is also a large continuum caused by deexcitation cascades via different states between the ground stated and starting level. As it is known from other experiments, most of the deexcitation will follow the way of the cascade because the probability for a direct ground state transition is low.

A new code has been developed, which calculates the deexcitation of the nucleus after the capture. This code is in principle similar to the idea from ref. [14]. But in difference to this we use a extreme statistical approach. Going away from calculating a complete level scheme we handled the deexcitation in energy bins of $10 \mathrm{keV}$. This could be justified with the large number of levels at high energies and 


\section{EPJ Web of Conferences}

under the assumption, that level density and strength function change smoothly between the chosen energy steps. The advantage is a very fast calculation by using the following assumptions:

- the neutrons are captured as s-wave neutrons. This implies a mixture of the start level with spin 0 and 1

- usage of E1, M1 and E2 transitions

- a parametrization of the E1-GDR with three Lorentzian curves, cf. fig. 1, which fits well the known $(\gamma, \mathrm{n})$-measurements

- parametrization for M1 following a globalized fit on the data published in ref. [15]

- parametrization of the E2 strength according to RIPL-3

- use of information discrete levels up to $2.5 \mathrm{MeV}$, beyond this a level density calculated with the constant temperature model [16]

- calculate spin distributions in level density according to ref. [17]

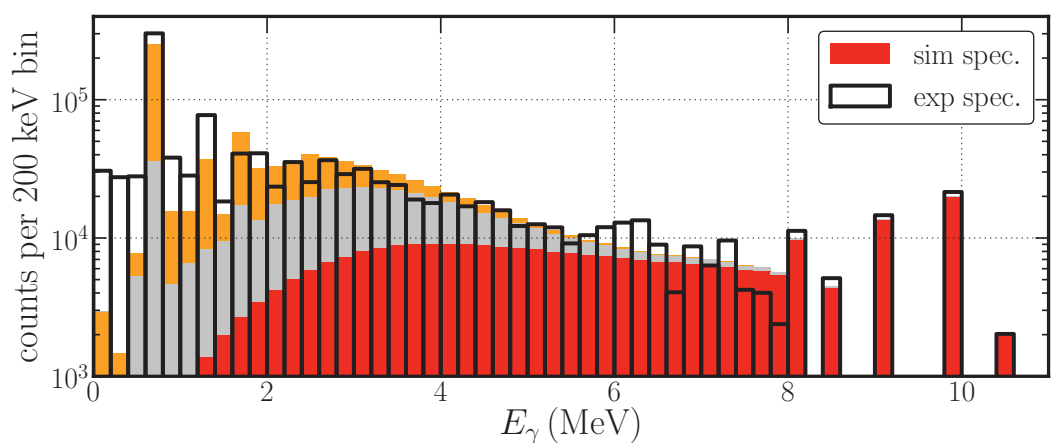

Fig. 4. comparison of experimental results and simulation. The different colors show the different generations of photons. Red are primary gammas which are sent out directly from the start level. The gray bars show the secondary gammas, orange bars are higher order photons.

As fig. 4 shows it is possible to reproduce the experimental spectrum with this code. The speed of the calculation allows us to test the different input parameters.

As a final result we were able to to estimate a temperature of $T=900 \mathrm{keV}$ for the Constant Temperature model level density. This is in good agreement with other data where temperatures of $850 \mathrm{keV}$ [16] or $890 \mathrm{keV} \mathrm{[17]} \mathrm{are} \mathrm{predicted.} \mathrm{With} \mathrm{the} \mathrm{code} \mathrm{one} \mathrm{could} \mathrm{estimate} \mathrm{an} \mathrm{average} \mathrm{photon-multiplicity}$ per decay of approx. 3.6. This value could be used to calculate at $S_{n}$ the neutron capture cross section of $\sigma_{\gamma}=36(6) \mathrm{b}$. This also fits to other data for example at ref. [18] where the value is given by $\sigma_{\gamma}=42(4) \mathrm{b}$.

\section{$3(\gamma, \gamma)$ at ELBE in Dresden}

The bremsstrahlung facility at the ELBE accelerator ( superconducting Electron Linac with high Brilliance and low Emittance ) of the Helmholtz-Zentrum Dresden-Rossendorf opens up the possibility to investigate dipole strength in nuclei up to neutron separation energy. Bremsstrahlung is produced by electrons hitting a thin niobium foil with an tunable energy up to $18 \mathrm{MeV}$.

The neutron separation energy of the nucleus ${ }^{78}$ is at $10.498 \mathrm{MeV}$ so an electron energy of $11.5 \mathrm{MeV}$ was chosen to ensure a photon flux up to the opening $(\gamma, n)$ channel. The collimated photon beam impinged onto the target at a spot of about $38 \mathrm{~mm}$ in diameter. The target itself consists of $99.39 \%$ of enriched ${ }^{78} \mathrm{Se}$ and was pressed to a disk with a mass of $2 \mathrm{~g}$. In front of the target disk a small disk of ${ }^{11} \mathrm{~B}$ was placed with an amount of $0.328 \mathrm{~g}$. This target is used as a flux monitor because the scattering cross section for the single levels in ${ }^{11} \mathrm{~B}$ is well known. 


\section{CNR*11}

The scattered photons were measured in a setup which is built up by four high-purity germanium detectors (HPGe) with an relative efficiency of $100 \%$ in comparison to an NaI detector with a size of 3 in. in diameter and 3 in. in length. The HPGe are surrounded by bismuth-germanate scintillation detectors which are used a veto detectors to suppress Compton scattered background in the final spectra. In front of the detectors lead collimators with a thickness of $10 \mathrm{~cm}$ are situated.

One pair of detectors is placed under $90^{\circ}$ in a distance of $28 \mathrm{~cm}$ from the target. The other one is placed under $127^{\circ} 32 \mathrm{~cm}$ away from the target position. This configuration allows us to distinguish between dipole an quadrupole transitions.

The energy-integrated scattering cross section $I_{s}$ of an excited state at the energy $E_{x}$ could be calculated from the measured intensity of the respective transition to the ground state (elastic scattering). It can be determined relative to the known integrated scattering cross sections $I_{s}\left(E_{x}^{\mathrm{B}}\right)$ of states in ${ }^{11} \mathrm{~B}$ [19]:

$$
\frac{I_{s}\left(E_{x}\right)}{I_{s}\left(E_{x}^{\mathrm{B}}\right)}=\left(\frac{I_{\gamma}\left(E_{\gamma}, \theta\right)}{W\left(E_{\gamma}, \theta\right) \Phi_{\gamma}\left(E_{x}\right) N_{N}}\right)\left(\frac{I_{\gamma}\left(E_{\gamma}^{\mathrm{B}}, \theta\right)}{W\left(E_{\gamma}^{\mathrm{B}}, \theta\right) \Phi_{\gamma}\left(E_{x}^{\mathrm{B}}\right) N_{N}^{\mathrm{B}}}\right)^{-1} .
$$

Here, $I_{\gamma}\left(E_{\gamma}, \theta\right)$ and $I_{\gamma}\left(E_{\gamma}^{\mathrm{B}}, \theta\right)$ denote the measured intensities of a considered ground-state transition at $E_{\gamma}$ and of a ground-state transition in ${ }^{11} \mathrm{~B}$ at $E_{\gamma}^{\mathrm{B}}$, respectively, observed at an angle $\theta$ to the beam. $W\left(E_{\gamma}, \theta\right)$ and $W\left(E_{\gamma}^{\mathrm{B}}, \theta\right)$ describe the angular correlations of these transitions. The quantities $N_{N}$ and $N_{N}^{\mathrm{B}}$ are the numbers of nuclei in the ${ }^{78} \mathrm{Sr}$ and ${ }^{11} \mathrm{~B}$ targets. The quantities $\Phi_{\gamma}\left(E_{x}\right)$ and $\Phi_{\gamma}\left(E_{x}^{\mathrm{B}}\right)$ stand for the photon fluxes at the energy of the considered level and at the energy of a level in ${ }^{11} \mathrm{~B}$.

The calculation of the complete photo absorption cross section is only possible after several corrections of the measured spectrum [20]. At first, one has to correct for detector response and efficiency. As well as in Budapest the geometry and setup are placed in a GEANT4 simulation and calculated with an very well agreement. After this correction also the influence of not nuclear scattered events is determined by another GEANT4 simulation which is adjusted to the spectra by the total flux.

As it could be seen in fig. 5 a large continuum is left over the atomic background. This continuum contains some strong peaks, as well as a large amount of unresolved peaks. By doing the following steps in a bin-wise work is possible to take account about this continuum in difference to other works performed in the field of nuclear resonance fluorescence.

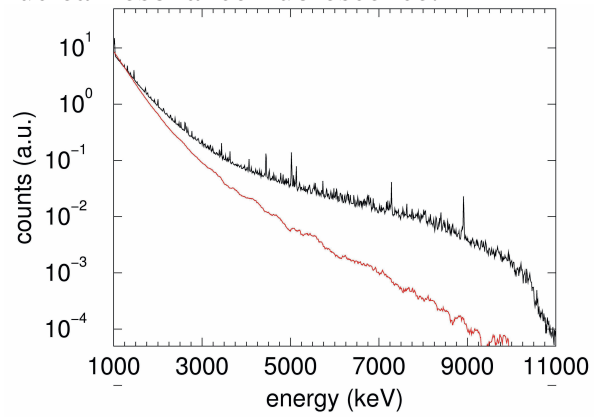

Fig. 5. simulation of the atomic background (red) and experimental spectrum (black) for the two detectors under $127^{\circ}$

The last step in analysis is to remove the inelastic transitions from the spectrum and correct the observed ground state transitions with the branching ratio. Both steps are done by a statistical calculation. The average branching ratio in one bin as well as the deexcitation spectra for the excited states in this bin are simulated by using the input parameters mentioned earlier in chapter 2.2. One big difference to the deexcitation of a state excited by neutron capture is that one has to take care about the so called enhancement. In fact one has to consider that in the method of photon excitation one prefers the excitation of levels with a large ground state width. When once excited these levels will decay with high probability directly back to the ground state. This misaligns the average ground state ratio to the 
one, one would expect by just using the simple approach with strength function and level densities. In ref. $[11,12]$ it is shown how this relocation changes with the excitation energy.
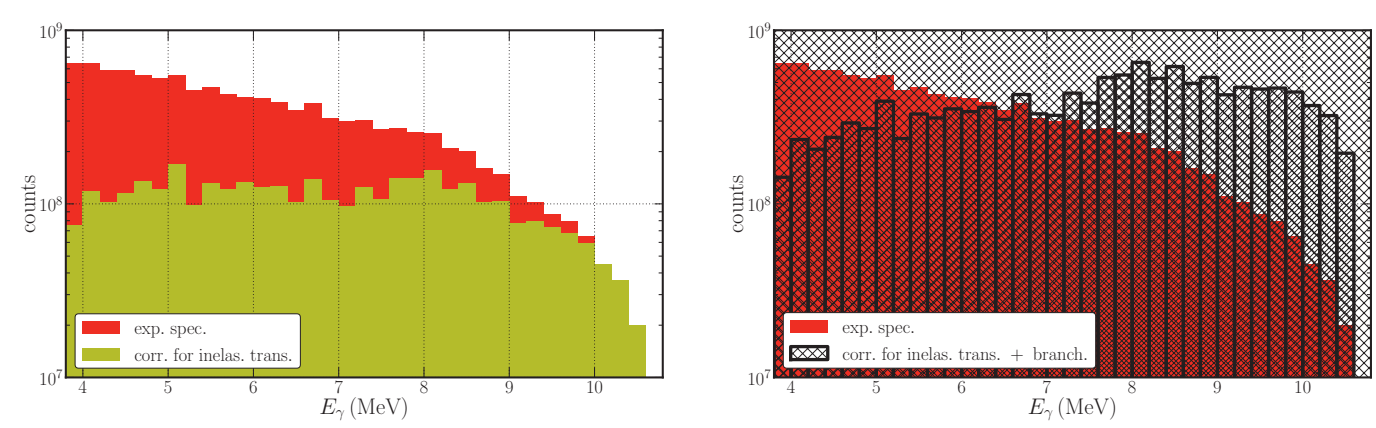

Fig. 6. (left) The inelastic transition are removed from the spectra (red) which was corrected for detector response as well as for natural and atomic background. The leftover (yellow) represents the observed elastic transitions in the experiment. (right) The elastic part is moved up by using the calculated branching ratio (black).

As a final result we were able to calculate the photo-absorption cross section up to neutron separation energy. The data fits well to data from $(\gamma, \mathrm{n})$ experiments but in fact it is observable that one could not describe the low energy part of the GDR with the tail of one or more Lorentzian curves due to the observed extra strength. This additional strength is located at around 8 to $9 \mathrm{MeV}$ and the excess could be estimated to approximately $2 \%$ of the Thomas-Reiche-Kuhn (TRK) sum rule.

The experiments were supported by the transnational access activity of the EURATOM FP6 project EFNUDAT (036434).

\section{References}

1. R. Capote et al. Nuclear Data Sheets 1103107 (2009)

2. A.R. Junghans et al. Phys let. B 670, 200 (2008)

3. K. Langanke and M. Wiescher Rep.Prog. Phys 64, 1657 (2001)

4. W. Kleinig et al, Phys. Rev C 78, 044313 (2008)

5. A.M. Goryachev et al, J,VTYF 8,121 (1982)

6. R. Schwengner et al. Phys. Rev. C 78, 064314 (2008)

7. A. Zilges et al. Phys. Lett. B 542, 43 (2002)

8. S. Volz et al. Nucl. Phys. A779, 1 (2006)

9. R. Schwengner et al. Nucl. Instrum. Methods A 555 ,211 (2005)

10. S. Agostinelli et al , Nucl. Instrum. Methods A 506, 250 (2003)

11. G. Schramm Diploma thesis, Technical University Dresden (2011)

12. G. Schramm et al. Phys. Rev. C submitted (2011)

13. Zs. Révay et al. Nucl. Instrum. Methods B 213 ,385 (2004)

14. F. Bečvár Nucl. Instrum. Methods A 417,434 (1998)

15. K. Heyde et al. Rev.Mod. Phys. 82, 2365 (2010).

16. A. Koning et al. Nucl. Phys. A 810, 13 (2008)

17. T. von Egidy et al. Phys. Rev. C 80, 054310 (2009) 


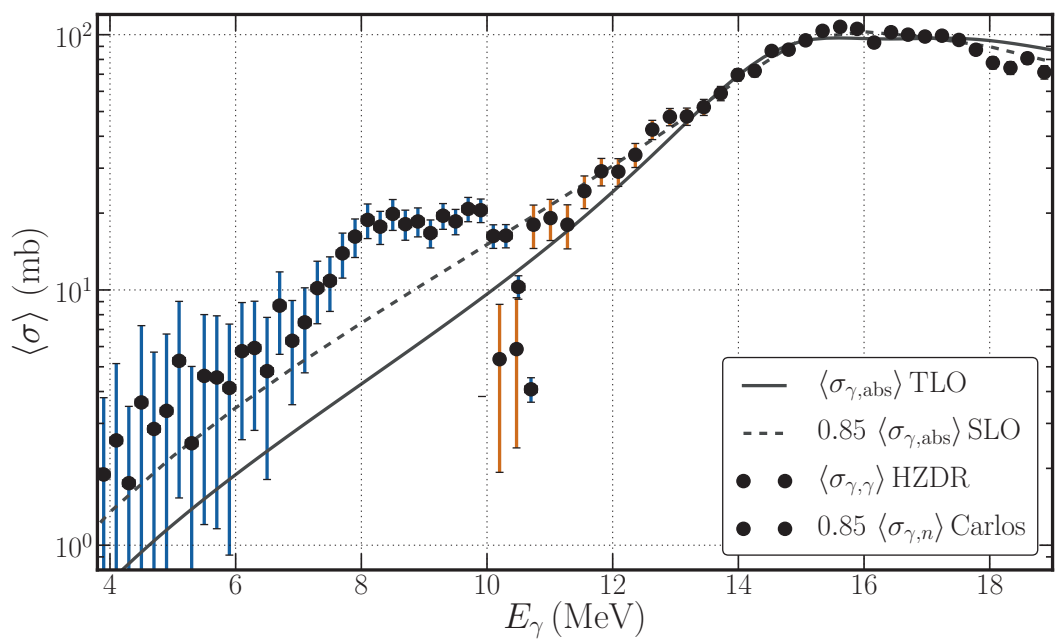

Fig. 7. Photo absorption cross section for ${ }^{78} \mathrm{Se}$. The final result fits well to $(\gamma, \mathrm{n})$ data and show an enhancement of about $2 \%$ relating to the TRK sum rule.

18. S. Mughabghab, Atlas of neutron resonances, Elsevier vol. 5 (2006)

19. F. Ajzenberg-Selove and J. H. Kelley, Nucl. Phys. A506,1 (1990).

20. R. Massarczyk Diploma thesis, Technical University Dresden (2011) 